\title{
Scandinavian Journal of Gastroenterology
}

\section{Super selective arterial embolization to treat radiation-induced hemorrhagic gastritis: a case report and review of the literature}

\author{
Michelangela Mossa, Benedetto Neri , Patrizio Scarozza, Giovanna Del \\ Vecchio Blanco, Mario Giannelli , Renato Argirò , Sara Di Carlo , Giovanni \\ Monteleone \& Carmelina Petruzziello
}

To cite this article: Michelangela Mossa , Benedetto Neri , Patrizio Scarozza , Giovanna Del Vecchio Blanco, Mario Giannelli , Renato Argirò , Sara Di Carlo, Giovanni Monteleone \& Carmelina Petruzziello (2020): Super selective arterial embolization to treat radiation-induced hemorrhagic gastritis: a case report and review of the literature, Scandinavian Journal of Gastroenterology, DOI: 10.1080/00365521.2020.1853219

To link to this article: https://doi.org/10.1080/00365521.2020.1853219

\section{册 Published online: 02 Dec 2020.}

Submit your article to this journal $\pi$

\section{Џ Article views: 2}

a)

View related articles

\section{View Crossmark data $[7$}




\title{
Super selective arterial embolization to treat radiation-induced hemorrhagic gastritis: a case report and review of the literature
}

\author{
Michelangela Mossa ${ }^{a}$ (D), Benedetto Neri ${ }^{\mathrm{a}}$ (D), Patrizio Scarozza ${ }^{\mathrm{a}}$, Giovanna Del Vecchio Blanco ${ }^{\mathrm{a}}$, Mario Giannelli ${ }^{\mathrm{a}}$ \\ Renato Argirò ${ }^{b}$, Sara Di Carloc, Giovanni Monteleone ${ }^{a}$ and Carmelina Petruzziello ${ }^{a}$ \\ aDepartment of Systems Medicine, GI Unit, University "Tor Vergata" of Rome, Rome, Italy; ${ }^{\text {b} D e p a r t m e n t ~ o f ~ D i a g n o s t i c ~ a n d ~ I n t e r v e n t i o n a l ~}$ \\ Radiology, University "Tor Vergata" of Rome, Rome, Italy; 'Department of Surgery, Minimally Invasive and Gl Surgery, University \\ "Tor Vergata" of Rome, Rome, Italy
}

\begin{abstract}
Radiation-induced hemorrhagic gastritis (RIHG) is a rare but potentially fatal event following radiotherapy for locally advanced gastric cancer; the treatment of this condition is not standardized. Only few cases of RIHG have been reported, treated with different therapeutic approaches. Here we report the case of a 79-year-old patient who underwent subtotal gastrectomy for gastric cancer, followed by adjuvant chemo-radiotherapy. Approximately 3 months after the end of the treatment, she developed recurrent diffuse bleeding originating from the entire mucosa of the gastric pouch and from a marginal ulcer. As the bleeding was refractory to several endoscopic treatments and surgery was not indicated, the patient underwent two sessions of transcatheter selective arterial embolization, with resolution of bleeding. Arterial embolization has already been reported for the treatment of hemorrhagic cystitis, developing after irradiation of the pelvis for prostate, bladder, rectum, and cervix cancer. However, to our knowledge, it has never been reported as a treatment for hemorrhagic gastritis. Based on this case, we suggest arterial embolization as an option in the management of RIHG, when standard endoscopic treatment fails.
\end{abstract}

\section{ARTICLE HISTORY}

Received 19 August 2020

Accepted 12 November 2020

\section{KEYWORDS}

Gastric cancer; radiotherapy; radiation-induced hemorrhagic gastritis; APC; transcatheter arterial embolization

\section{Introduction}

Gastric cancer is the fifth most frequent cancer and the third-leading cause of cancer related death [1]. Surgical resection is potentially curative at early stages, moreover, for locally advanced cancer combined chemo-radiotherapy is recommended to prevent the risk of relapse [2]. Radiation therapy can be complicated by either acute or late gastric toxicity, related to total dose of radiation, fraction size and volume irradiated $[3,4]$. After irradiation, nausea and vomiting can occur within few hours while ulceration and bleeding can appear after several days or weeks. Hemorrhagic gastritis is a rare complication following radiotherapy and it is a lifethreatening issue for which there is no standard therapy [5]. Here we report a case of recurrent and diffuse bleeding due to radiation-induced gastritis, refractory to multiple endoscopic therapies and successfully controlled by super selective transcatheter arterial embolization (TAE).

\section{Case Presentation}

In January 2019, a 79-year-old housewife, affected by permanent atrial fibrillation on Warfarin anticoagulant therapy, with mitral and aortic mechanical valves, was admitted to our hospital for the progressive development of asthenia and the detection of severe iron deficiency anemia $(\mathrm{Hb} 4 \mathrm{~g} /$ dl). She was diagnosed with locally advanced gastric adenocarcinoma ( $\mathrm{pT} 4, \mathrm{~N} 0, \mathrm{M} 0$ ) and 1 month after, she underwent subtotal gastrectomy with Roux-en-Y reconstruction. Capecitabine systemic chemotherapy was administered concurrently with radiation therapy. From 11 July to 16 August 2019, the patient underwent 25 sessions of radiotherapy receiving a total dosage of $45 \mathrm{~Gy}$ and a daily dosage of $1.8 \mathrm{~Gy}$. The last chemotherapy cycle ended on 23 October 2019. In November 2019 (80 days after completing radiotherapy), a routine blood test revealed severe normochromic normocytic anemia $(\mathrm{Hb} 6 \mathrm{~g} / \mathrm{dl})$, with no signs of gastrointestinal bleeding (GIB). The patient was admitted to the emergency department where she was transfused with 2 units of packed red blood cells (RBC) while oral anticoagulant therapy was shifted to subcutaneous Enoxaparin. She was then discharged with a $\mathrm{Hb}$ level of $9 \mathrm{~g} / \mathrm{dl}$ with indication for outpatient esophagogastroduodenoscopy (EGD). One week after the discharge, the patient underwent EGD showing a single ulcer with adherent clot at the gastrojejunal anastomosis and a large non-aspirable clot in the gastric fundus; no treatment was performed. She was admitted to the emergency department where she underwent an urgent abdominal contrast-enhanced CT scan, negative for active bleeding. During the hospitalization in the Gastroenterology Unit, she underwent EGD with biopsies taken from the anastomothic 
mucosa which ruled-out cancer recurrence. Intravenous highdose proton pump inhibitor (PPI) was administered and 2 units of packed RBC were transfused. The patient was discharged with a Hb level of $9.1 \mathrm{~g} / \mathrm{dl}$. In December 2019, 2 days after reintroducing oral anticoagulant therapy with Warfarin, the patient was readmitted to the emergency department for coffee ground emesis. Hb detected was $9.7 \mathrm{~g} / \mathrm{dl}$, INR 3.69; oral anticoagulant therapy was shifted again to Enoxaparin and high-dose PPI was administered. The patient underwent to EGD showing a small Forrest III ulcer on the gastrojejunal anastomosis and active bleeding coming from the entire mucosa of the gastric pouch; the oozing area was so large that, therefore, a treatment with Hemospray (Cook Medical-Hemospray Endoscopic Hemostat) was done. Taken in account the clinical history of the patient, the absence of cancer recurrence and the endoscopic findings, a diagnosis of radiation-induced hemorrhagic gastritis (RIHG) was made. Two weeks later, due to a new episode of hematemesis, an emergency EGD was performed, showing a friable and hyperemic mucosa of the anastomosis, covered by a large clot with underlying oozing hemorrhage, requiring two sessions of APC (APC probe; ERBE Elektromedizin, Tubingen, Germany). APC was also performed on the entire oozing mucosa of the pouch. After a multidisciplinary team discussion, weighting risks and benefits of available treatments, a conservative management was established. On January 2020, after several RBC transfusions, the patient was re-evaluated through EGD, that still showed diffuse bleeding from the entire mucosa of the gastric pouch (Figure 1(A)); APC and 3-clips placing (Cook Medical-Instinct Endoscopic Hemoclip) on the anastomosis were performed (Figure 1(B)). As active bleeding persisted, a treatment with Hemospray was attempted, without success (Figure 1(C)). On the same day, selective angiography with right femoral access with a $5 \mathrm{Fr}$ catheter (Simmonds Glidecat Terumo) at the level of the celiac axis was performed. Since then the patient had been transfused with a total of 14 units of packed RBC. The angiography showed small angiectasias and hypervascularization of the upper portion of the gastric pouch from the left gastric artery (Figure 2(A)). Selective evaluation of splenic artery demonstrated other areas of hypervascularization for the inferior part of the gastric pouch with direct vascularization from a hypertrophic short gastric artery and a tortuous collateral circle arising from the inferior lobar branch of the splenic artery. A super selective catheterization of the left gastric artery with $2.7 \mathrm{Fr}$ microcatheter (Progreat, Terumo) was performed with subsequent embolization with particles of polyvinyl alcohol (Contour 250-350 micron particles, Boston Scientific) (Figure 2(B)); the embolization of the other gastric arterial "feeders" was considered too risky to be performed in a single session. In February 2020, after 1 week of clinical observation, the patient was discharged. Three weeks after the discharge, due to the recurrence of bleeding, a selective catheterization of the splenic artery was performed confirming the tortuous collateral vascular circle directed to the gastro-jejunal anastomosis (Figure 2(C)). With a $2.7 \mathrm{Fr}$ catheter, this artery was super selectively catheterized and embolized initially with particles of polyvinyl alcohol (Contour 250-350 micron particles, Boston Scientific, Marlborough, MA) and with $3 \mathrm{~mm}$ pushable coils (VortX 0,0018", Boston Scientific, Marlborough, MA) (Figure 2(D)). Subsequently, a super selective catheterization and embolization of the hypertrophic short gastric artery were performed (Figure 2(E,F)); at the end of the procedure, the angiography from the celiac axis documented the presence of another collateral circle arising from the left inferior phrenic artery that was embolized with the same particles (Figure $2(\mathrm{G}, \mathrm{H})$ ). Six days after the procedure, on March, the patient was discharged. The EGDS performed 4 months later, on July 2020, showed only a slight hyperemia in the cardias; the remaining mucosa of the pouch and of the gastrojejunal anastomosis was regular and healthy (Figure 1(D)).

\section{Discussion}

Radiation-induced hemorrhagic gastritis (RIHG) is an uncommon event that may complicate radiotherapy, leading to a severe GIB which makes it a potentially life-threatening complication. Radiations induce acute vasculopathy which progresses to obliterative endarteritis. This could lead either to mucosal ischemia with ulceration or to endothelial proliferation with the development of telangiectasias with subsequent bleeding [5]. The endoscopic findings of RIHG include diffusely friable, granular and hyperemic mucosa, multiple hemorrhagic patches and petechiae in the acute period (within 90 days from radiotherapy) while telangiectasias, shallow or deep ulcers and scars are more frequent in the late period (after 90 days) [6]. According to Emami et al., the TD $5 / 5$ of the stomach (dose tolerance with a $5 \%$ risk of complication within five years from treatment) for ulceration-perforation, is $60 \mathrm{~Gy}, 55 \mathrm{~Gy}$, and $50 \mathrm{~Gy}$ when one-third, two-thirds and, the entire stomach is irradiated, respectively [7]. Late effects (primarily ulceration) occur approximately in 5\% to $7 \%$ of cases when the whole stomach is irradiated by doses of radiation around $45 \mathrm{~Gy}$. A larger fraction size and a higher total dose are related to an increased rate of complications and a higher volume irradiated reduces the tolerance dose $[3,4]$. Since RIHG is a rare complication following radiotherapy, its management is not well defined. Staiano et al. first described a case of a 69-year-old woman who underwent Billroth II gastrojejunostomy plus adjuvant chemoradiation for locally advanced gastric adenocarcinoma. The patient developed 6 months later a RIHG, successfully treated with three sessions of endoscopic band ligation [8]. Morrow et al. successfully managed a case of RIHG limited to the region of the cardia by APC [9]. Combined therapy with APC and radiofrequency ablation has been described by Ross et al. for the treatment of diffuse RIHG [10]. Further potential treatment modality have been reported, particularly when endoscopic coagulation therapy is not effective: hyperbaric oxygen treatment, growth hormone and steroid therapy either as steroid infusions directly administered in the right gastroepiploic artery, either as oral therapy - have also been described as effective in the treatment of refractory postradiation gastric bleeding [5,11-13]. Bevacizumab, a fully human monoclonal antibody directed towards VEGF, resulted 


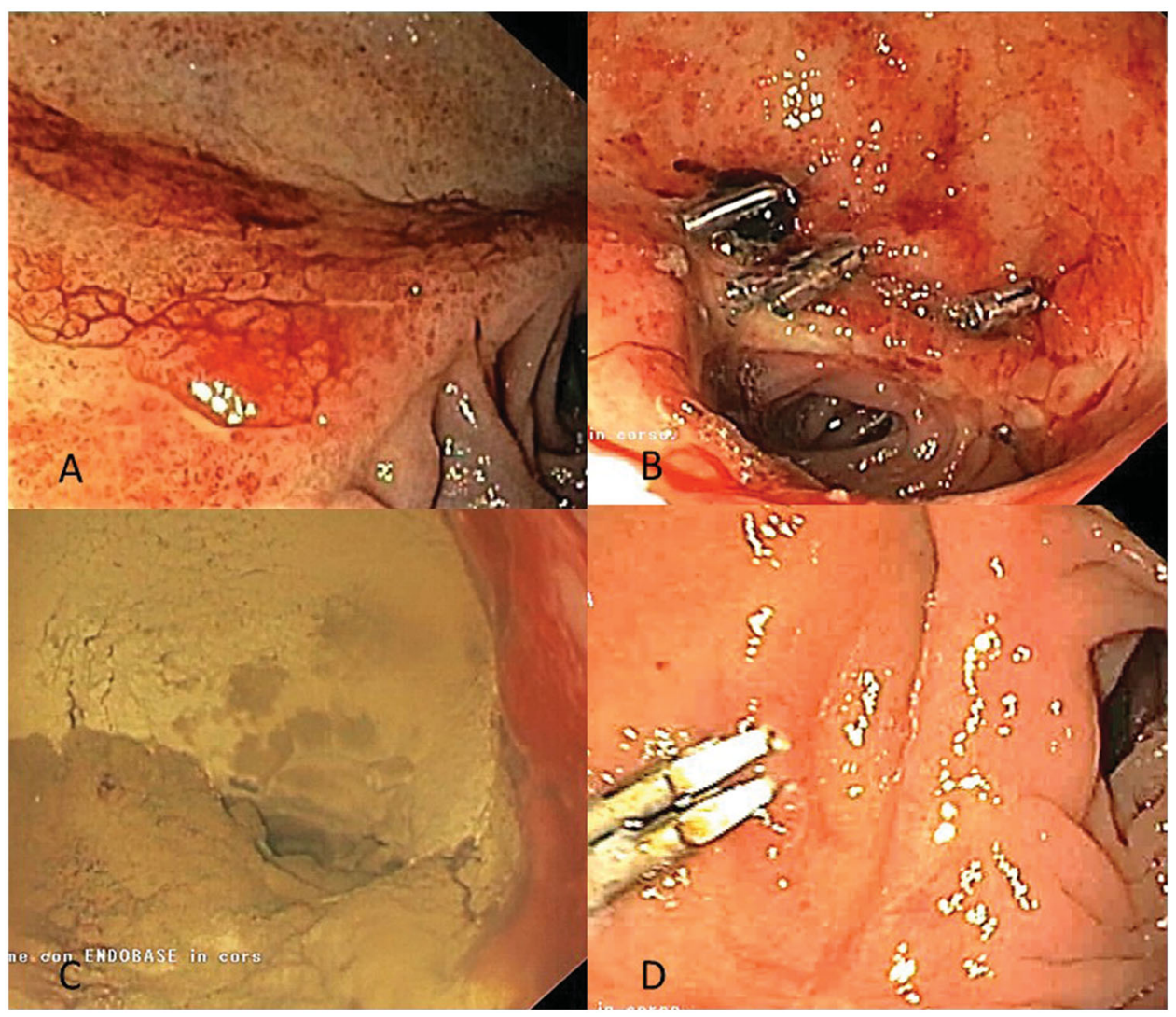

Figure 1. (A-D). Endoscopic image of the oozing bleeding coming from the hyperemic, friable and ulcerated mucosa of the gastric pouch (A); endoscopic image of clips placed on the gastrojejunal anastomosis (B); endoscopic image showing the use of Hemospray to treat the oozing mucosa (C); endoscopic image showing the healthy mucosa of the gastric pouch and the gastrojejunal anastomosis, 4 months after arterial embolization (D).

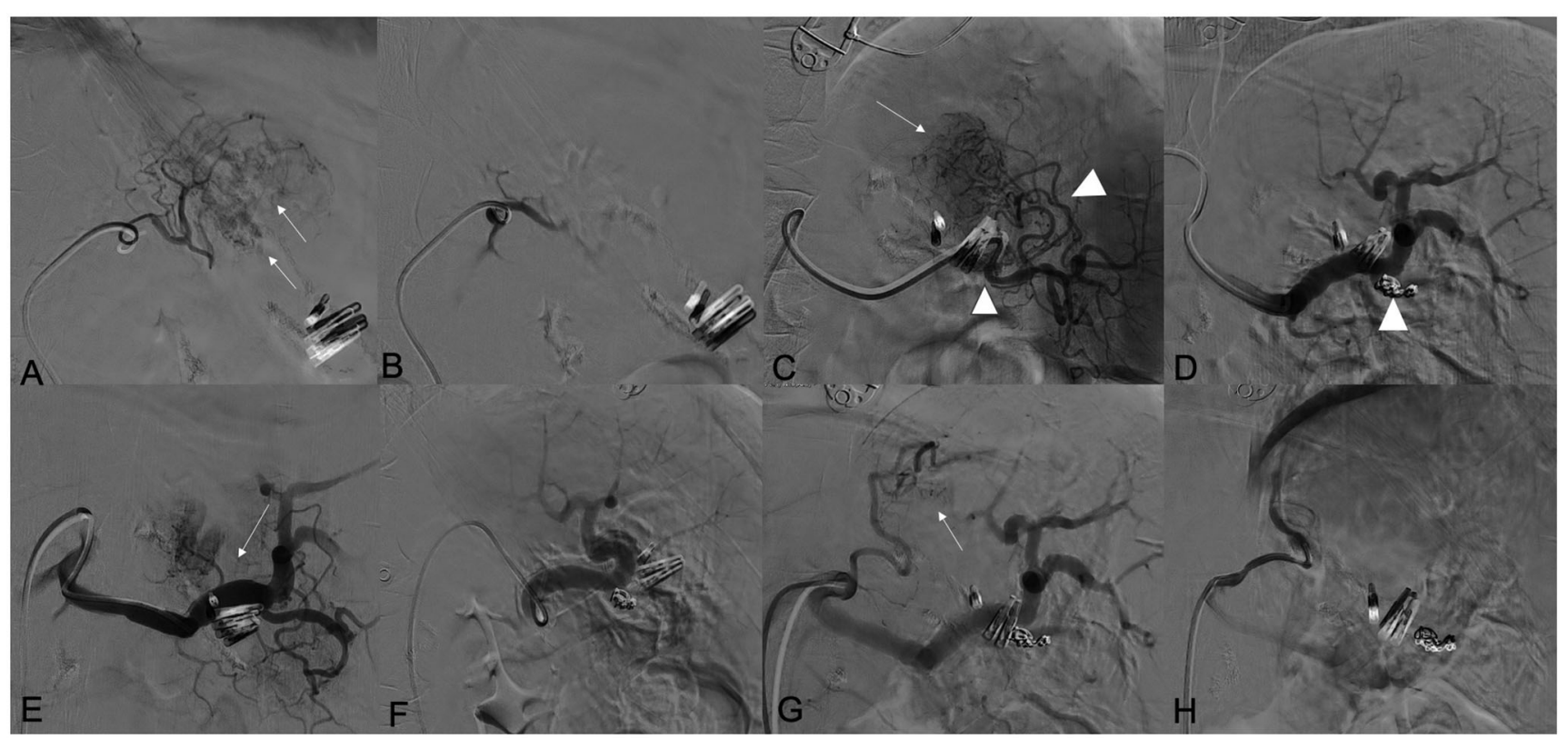

Figure 2. (A-H). Fluoroscopic frames of selective catheterization of left gastric artery performed with $5 \mathrm{Fr}$ catheter; angiography demonstrates small angiectasias and hypervascularization of the upper portion of gastric pouch (arrows) (A); angiography obtained after embolization with no residual direct opacification of the upper gastric pouch (B); selective angiography of distal splenic artery demonstrates presence of a tortuous collateral circle (arrowhead) with direct vascularization of the lower portion of the gastric pouch (arrow) (C); results after the embolization with coils (arrowhead) of the collateral circle with no evidence of direct opacification of gastric pouch (D); angiography of the whole splenic artery with opacification of a hypervascular portion of the gastric pouch from a short gastric artery (arrow) (E); results after embolization without absence of arterial enhancement (F); superselective catheterization of the left lower phrenic artery with evidence of small tortuous vessels at the level of the upper gastric pouch (arrow) $(G)$ with results after the embolization (H). 
effective in the setting of recurrent bleeding due to radiation gastroduodenal telangiectasias [14]. When hemorrhagic gastritis occurs as a severe recurrent bleeding not responsive to standard endoscopic treatments, surgery may represent a valid therapeutic option. However, few cases treated with surgery are reported $[15,16]$. Recently, Tatsis et al. described the case of a 56-year-old man with hilar cholangiocarcinoma, developing upper GIB 3 months after the last session of radiotherapy. After several attempts of endoscopic hemostasis, the patient underwent subtotal gastrectomy with Rouxen-Y reconstruction, which brought to case resolution [17]. In our patient, hemorrhagic gastritis developed 3 months after completing radiotherapy, presenting both as diffuse oozing from the widely friable mucosa of the pouch and as anastomotic ulcer. The bleeding from the anastomosis was finally and successfully managed with clip placing. The oozing area of the pouch was so diffuse that at first we tried to achieve hemostasis using hemostatic power (Hemospray) without success. Despite the extension of the injured gastric mucosa was a limit for the use of endoscopic electrocoagulation therapy, an effort was made to control the bleeding with APC. The failure of all the proposed approaches forced us to consider other therapeutic options. Surgery was excluded because converting subtotal gastrectomy into total gastrectomy in an old patient with multiple comorbidities was burdened by a too high risk of complications. Now-a-days, due to the evolution of interventional radiology and to the technological improvements, TAE has become a valid low invasive method alternative to surgery in the management of upper GIB. In a recent meta-analysis, it was shown that TAE is an effective procedure in controlling GIB, but with a higher rebleeding rate compared to surgery, even though without apparent impact on clinical outcome [18]. However, in the absence of evident superiority of a specific treatment, patients' background and local expertise continue to drive therapeutic choices. The use of arterial embolization for the management of recurrent bleeding occurring as a complication of radiotherapy has already been reported for radiation proctitis and hemorrhagic cystitis developing after irradiation of the pelvis for prostate, bladder, rectum, and cervix cancer $[19,20]$. However, to our knowledge, it has not been reported in the field of hemorrhagic gastritis. Therefore, this is the first reported case of radiation-induced hemorrhagic gastritis refractory to several endoscopic treatments in a patient underwent subtotal gastrectomy for gastric cancer, successfully treated by super selective transcatheter arterial embolization.

\section{Disclosure statement}

The authors report no conflict of interest.

\section{ORCID}

Michelangela Mossa (iD http://orcid.org/0000-0001-5013-7484 Benedetto Neri (ID) http://orcid.org/0000-0002-0803-7903

\section{References}

[1] Arnold M, Abnet CC, Neale RE, et al. Global burden of 5 major types of gastrointestinal cancer. Gastroenterology. 2020;159(1): 335-349.e15.

[2] Smyth EC, Verheij M, Allum W, et al. Gastric cancer: ESMO Clinical Practice Guidelines for diagnosis, treatment and follow-up. Ann Oncol. 2016;27(5):v38-v49.

[3] Coia LR, Myerson RJ, Tepper JE. Late effects of radiation therapy on the gastrointestinal tract. Int J Radiat Oncol Biol Phys. 1995; 31(5):1213-1236.

[4] Kavanagh BD, Pan CC, Dawson LA, et al. Radiation dose-volume effects in the stomach and small bowel. Int J Radiat Oncol Biol Phys. 2010;76(3):101-107.

[5] Zhang L, Xie XY, Wang $Y$, et al. Treatment of radiation-induced hemorrhagic gastritis with prednisolone: a case report. World J Gastroenterol. 2012;18(48):7402-7404.

[6] Chao CJ, Shin JS, Hsu WC, et al. Endoscopic features of radiation gastritis after irradiation of hepatocellular carcinoma. Endoscopy. 2013;45*(S 02):E280-E281.

[7] Emami B, Lyman J, Brown A, et al. Tolerance of normal tissue to therapeutic irradiation. Int J Radiat Oncol Biol Phys. 1991;21(1): 109-122.

[8] Staiano T, Grassia R, liritano E, et al. Treatment of radiationinduced hemorrhagic gastritis with endoscopic band ligation. Gastrointest Endosc. 2010;72(2):452-453.

[9] Morrow JB, Dumot JA, Vargo JJ. Radiation-induced hemorrhagic carditis treated with argon plasma coagulator. Gastrointest Endosc. 2000;51(4 Pt 1):498-499.

[10] Ross A, Kuppusamy M, Low D. Endoscopic management of postesophagectomy hemorrhagic radiation gastritis with radiofrequency ablation and argon plasma coagulation. Gastrointest Endosc. 2012;75(6):1285-1286.

[11] Kernstine $\mathrm{KH}$, Greensmith JE, Johlin FC, et al. Hyperbaric oxygen treatment of hemorrhagic radiation-induced gastritis after esophagectomy. Ann Thorac Surg. 2005;80(3):1115-1117.

[12] Inaba K, Sakurai Y, Furuta S, et al. Hemorrhagic radiation gastritis after chemoradiation therapy for peritoneal lymphnode metastasis of esophageal carcinoma successfully treated with repeated intraarterial steroid infusions. Esophagus. 2007;4(2):67-72.

[13] Zhang L, Xia WJ, Zhang ZS, et al. Growth hormone used to control intractable bleeding caused by radiation-induced gastritis. World J Gastroenterol. 2015;21(31):9453-9456.

[14] Frédérique $M$, Muller $N$, Lévy $P$. First case of radiation-induced diffuse hemorrhagic gastritis successfully treated with intravenous bevazicumab. Am J Gastroenterol. 2017;112(8):1349-1350.

[15] Flobert C, Cellier C, Landi B, et al. Severe hemorrhagic gastritis of radiation origin. Gastroenterol Clin Biol. 1998;22(2):232-234.

[16] Yeung YP, Ho CM, Wong $\mathrm{KH}$, et al. Surgical treatment of recalcitrant radiation-induced gastric erosions. Head Neck. 2000;22(3): 303-306.

[17] Tatsis V, Peponi E, Papadopoulos G, et al. Subtotal gastrectomy for diffused hemorrhagic gastritis induced by radiation, following liver resection for hilar cholangiocarcinoma. A case report. Int J Surg Case Rep. 2016;18:30-32.

[18] Tarasconi A, Baiocchi GL, Pattonieri V, et al. Transcatheter arterial embolization versus surgery for refractory non-variceal upper gastrointestinal bleeding: a meta-analysis. World J Emerg Surg. 2019;14:3.

[19] Syed MI, Chaudhry N, Shaikh A, eta al. Catheter-directed middle hemorrhoidal artery embolization for life-threatening rectal bleeding. Can J Gastroenterol. 2007;21(2):117-123.

[20] De Berardinis E, Vicini P, Salvatori F, et al. Superselective embolization of bladder arteries in the treatment of intractable bladder haemorrhage. Int J Urol. 2005;12(5):503-505. 